\title{
Comparison of the Effects of Pioglitazone versus Placebo when Given in Addition to Standard Insulin Treatment in Patients with Type 2 Diabetes Mellitus Requiring Hemodialysis: Results from the PIOren Study
}

\author{
Jan Galle ${ }^{a}$ Werner Kleophas ${ }^{b}$ Frank Dellanna ${ }^{b}$ Volkmar H.R. Schmid ${ }^{c}$ \\ Claudia Forkel $^{c}$ Gerhard Dikta ${ }^{d}$ Vera Krajewski ${ }^{e}$ Winfried Fuchs ${ }^{e}$ \\ Thomas Forst ${ }^{f}$ Andreas Pfützner ${ }^{c, f}$ \\ ${ }^{a}$ Klinik für Nephrologie und Dialyseverfahren, Klinikum Lüdenscheid, Lüdenscheid, b Nephrology Practice, \\ Düsseldorf, cikfe CRO, Mainz, dUniversity of Applied Sciences, Julich, eTakeda Pharma GmbH, Aachen, and \\ $f_{\text {ikfe, Mainz, Germany }}$
}

\section{Key Words}

Glycemic control $\cdot$ Hemodialysis $\cdot$ Insulin reduction $\cdot$ Kidney failure $\cdot$ Pioglitazone $\cdot$ Type 2 diabetes mellitus

\begin{abstract}
Background: Patients with type 2 diabetes mellitus and advanced kidney disease are usually treated with insulin. However, the prolonged pharmacokinetic insulin profile in patients with delayed renal insulin elimination impairs a successful therapy. Due to its hepatic metabolism, pioglitazone is a potential candidate for additional administration. The aim of this study was to investigate the effect of pioglitazone versus placebo on total daily insulin requirements and several pleiotropic factors in type 2 diabetes patients requiring hemodialysis. Methods: The effect of pioglitazone ( $30 \mathrm{mg}$ ) versus placebo was explored in this prospective, randomized, double-blind parallel multicenter phase II study analyzing data from 36 patients with type 2 diabetes mellitus currently under hemodialysis ( 25 male, 11 female, aged $69.2 \pm 7.9$ years, baseline HbA1c $7.6 \pm 0.9 \%)$. The most important efficacy parameters collected before dialysis and after an overnight fast at baseline and after 6 months were: total daily insulin dose, HbA1c, fasting blood glucose, adiponectin, HDL, LDL, triglycerides, NT-proBNP, and ultrafiltrate volume. $\boldsymbol{R e}$ sults: Application of pioglitazone resulted in a significant decrease of the daily insulin dose by


$35 \%$ versus baseline (placebo: $-10 \%, n . s$.$) , improvement in \mathrm{HbA1c}(-0.60 \pm 0.87 \%, \mathrm{p}=0.015$; placebo: $0.21 \pm 1.1 \%$, n.s.) and adiponectin ( $7.33 \pm 4.80 \mathrm{mg} / \mathrm{l}, \mathrm{p}<0.001$; placebo: $-1.37 \pm 2.56$ $\mathrm{mg} / \mathrm{l}, \mathrm{n} . \mathrm{s}$.). Slight improvements or no changes were seen with fasting glucose, triglycerides, HDL, LDL and NT-proBNP. There was no indication of increased hypoglycemia risk and volume overload by the addition of pioglitazone. Conclusions: Addition of pioglitazone to insulin in patients with late-stage kidney failure requiring hemodialysis is a well-tolerated treatment option that improves glycemic control with simultaneous insulin-sparing potential.

Copyright $\odot 2012$ S. Karger AG, Basel

\section{Introduction}

Both type 1 and type 2 diabetes mellitus play a major role in the development of kidney failure and end stage renal disease (ESRD) [1]. The development and progression of diabetic nephropathy as well as any other diabetic end-organ damage can be avoided or delayed by a sufficient glycemic control defined by international guideline recommendations. Several antidiabetic drugs are currently used for this purpose [2, 3]. However, once renal failure has progressed to ESRD with the need for hemodialysis, most common oral antidiabetic drugs, including metformin, sulfonylurea drugs, GLP1 analogs, and DPPIV inhibitors are contraindicated [4]. Therefore, hemodialysis patients are currently treated with insulin or insulin analogs to control their blood sugar levels. The reduced glomerular filtration rate in renal failure that leads to accumulation of most of the oral drugs [5] is also responsible for a prolonged pharmacokinetic profile of insulin [6] so that the insulin dose and the scheduling will have to be adapted.

Besides the negative impact of inadequate glycemic control as a significant cardiovascular risk factor, the impaired kidney function results in increased oxidative stress and correspondingly increased cardiovascular risk, especially in patients requiring hemodialysis [7]. Several potential mechanisms may explain this increased cardiovascular risk. A frequent finding is coexistence of several other independent cardiovascular risk factors including dyslipidemia, hypertension, and smoking $[8,9]$. In addition, impaired kidney function is associated with elevated markers of inflammation and other putative risk factors for cardiovascular events [10, 11]. Therefore, a therapeutic strategy aiming at a sufficient glycemic control and reduced oxidative stress at the same time seems to be a reasonable approach to reduce the risk of short- and long-term consequences, especially the cardiovascular mortality of such patients.

In general, only drugs that are primarily metabolized and eliminated via the hepatic route are suitable in ESRD under hemodialysis. This is the case for the PPAR- $\gamma$ agonist pioglitazone (PIO), which is therefore approved for use in chronic renal failure patients [12]. Next to its hypoglycemic action through reduction of peripheral insulin resistance, PIO has been demonstrated to possess a variety of pleiotropic effects reducing cardiovascular risk, including improvement of hypertension, dyslipidemia, chronic systemic inflammation, platelet function, lipid tissue composition, and atherosclerosis [13-15]. Furthermore, in the outcome study PROactive, PIO significantly reduced cardiovascular endpoints [16-18]. In a subanalysis of the PROactive study, Schneider et al. [19] showed that especially patients with more severe degrees of kidney failure, as assessed by glomerular filtration rate, may benefit from treatment with PIO.

The purpose of this study was to investigate the influence of PIO added to insulin therapy on total insulin requirements and the overall risk profile in patients with ESRD undergoing hemodialysis. 


\section{Subjects and Methods}

This prospective, randomized, parallel, double-blind, placebo (PLA)-controlled multicenter phase II trial was approved by the responsible local ethics committees and carried out in accordance with the Helsinki Declaration of 1975 between 2008 and 2010. The study population consisted of inadequately controlled patients with type 2 diabetes requiring dialysis for ESRD. The patients were cared for in 12 centers in Germany. Inclusion criteria were type 2 diabetes mellitus, patient on insulin treatment for at least 3 months, age 30-80 years, $\mathrm{HbAlc} \geq 6.0 \%$ and $<10 \%$, patient on hemodialysis with or without residual excretion, and insulin dose $>20$ IU/day. Exclusion criteria were amongst others history of type 1 diabetes, acute infections, history of hypersensitivity to the study drugs or to drugs with similar chemical structures, history of severe or multiple allergies, progressive fatal disease other than kidney failure, history of significant cardiovascular (e.g., CHF NYHA stage III-IV), respiratory, gastrointestinal, hepatic (e.g., ALAT >2.5 times the normal reference range) or hematological disease, and treatment with thiazolidinediones within the past 3 months.

The primary endpoint of the study was the change in the daily insulin dose (basal and prandial) after 6 months of treatment with either PIO or PLA given in addition to insulin. The total daily insulin dose was defined as the mean out of the daily insulin dose on 3 consecutive days before the respective visits. Secondary efficacy endpoints were the number of patients with a reduction of the daily insulin dose of $\geq 30 \%$, various laboratory parameters such as $\mathrm{HbA1c}$, glucose, C-peptide, intact proinsulin, adiponectin, relaxin, fetuin A, carbonyl protein, angiotensin, high-sensitivity C-reactive protein (hs-CRP), calcification markers (MPO, matrix Gla protein), lipids (cholesterol, HDL, LDL, triglycerides), matrix metallopeptidase 9 (MMP-9), monocyte chemotactic protein-1 (MCP-1), soluble E-selectin, oxidized LDL (ox LDL), PIO in serum, intact parathyroid hormone (PTH), and N-terminal fragment of pro-brain natriuretic peptide (NT-proBNP). Laboratory efficacy parameters were measured using blood collected prior to dialysis at visit 2 (baseline), visit 5 (12 weeks later), and visit 7 (6 months later). The influence of the treatment on cardiac function was furthermore evaluated as the change in the ultrafiltrate volumes during the course of the study. Another objective was the safety surveillance including assessment of adverse events (AE) and safety laboratory parameters.

After written informed consent was obtained from each participant, patients were randomized to either receive an additional treatment with PIO $(1 \times 30 \mathrm{mg} /$ day at breakfast $)$ or PLA for 6 months. As depicted in figure 1, the trial consisted of a screening visit (Visit 1, V1), a randomization visit (baseline, V2), four treatment visits (V3-V6), and a final visit after 6 months of treatment (V7). Advice for insulin dosage adaptation and choice of insulin was at the discretion of the investigator. At the discretion of the investigator, the initial insulin dose was reduced by $10 \%$ at the randomization visit (V2). Insulin was titrated to target a fasting blood glucose level of $80-120 \mathrm{mg} / \mathrm{dl}$.

Patients were allowed to use any further concomitant medications that they required as far as they did not belong to those representing exclusion criteria. If medically acceptable, all concomitant medications had to be kept constant during the investigation.

\section{Laboratory Methods}

Glucose, creatinine, and lipids were determined by standard reference methods (COABS, Roche Diagnostics, Mannheim, Germany). Insulin and C-peptide were measured with chemiluminescence (Liaison, Byk-Sangtec, Neu-Isenburg, Germany). ELISA methods were used to assess MPO, matrix Gla protein, relaxin (all Immundiagnostik AG, Bensheim, Germany), intact proinsulin (TecoMedical, Sissach, Switzerland), angiotensin, NT-ProBNP, 
Fig. 1. Overview of the study design with two treatment arms. The chronological order of visits and their temporal spreading are depicted.

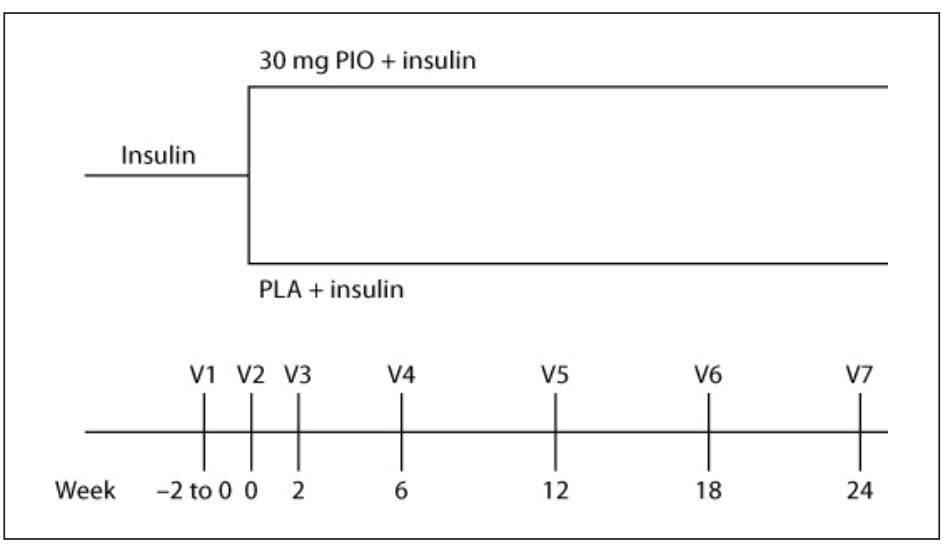

isoPGFa, MMP-9, MCP-1, and soluble E-selectin (all R\&D Systems, Hamburg, Germany). hs-CRP was assessed by turbidimetry (Falcor, Menarini, Neuss, Germany).

\section{Statistical Analysis}

The power calculation was based on the assumption that the required insulin dose would vary by $20 \%$ and the assumption that a decrease by $30 \%$ would be considered clinically relevant. Based on these conditions, the number per group required to have an $80 \%$ power $(\mathrm{a}=$ 0.05 ) for denying the appropriate null-hypothesis was 10 patients per group. As there was a high number of dropouts expected over the 6-month treatment period, the planned number of patients was 20 per treatment arm.

The primary efficacy variable was analyzed by descriptive statistics (mean, standard deviation, minimum value, 1 st quartile, median, 3rd quartile, maximum value) and inferential statistics [analysis of covariates (ANCOVA), Student's t test, Wilcoxon rank test, confidence intervals within treatment group and between treatment groups]. Secondary efficacy parameters were analyzed by the same descriptive statistics, by confidence intervals and two-sided Student's t tests.

The following analysis set was used: (1) intention-to-treat (ITT) set: all patients who had been randomized; (2) full analysis set (FAS): all randomized patients who received study medication and who provided one post-baseline value for the daily insulin dose. Primary and secondary efficacy variables were analyzed for the FAS. Demographic data are displayed for the FAS as well. The ITT set was used for evaluation of medical history, physical examination, concurrent diseases, concomitant medications, and safety issues (AEs, laboratory safety parameters). The analyses were performed by means of the SPSS software (version 16.0, SPSS Inc., Chicago, Ill., USA). A p value $<0.05$ was considered to be of statistical significance.

\section{Results}

The study was performed between August 2008 and June 2009. Screening of 52 patients in 14 study centers led to an ITT set of 39 patients aged between 50 and 80 years (69\% male). Three patients were erroneously randomized and therefore excluded prior to visit 3 as they did not meet all inclusion criteria. Therefore, the FAS consisted of 36 patients. Due to dropouts, the per protocol set (PPS) finally consisted of 26 patients, 15 belonging to the PIO group and 11 to the PLA group. Details about the reasons for dropout can be found in figure 2 . 


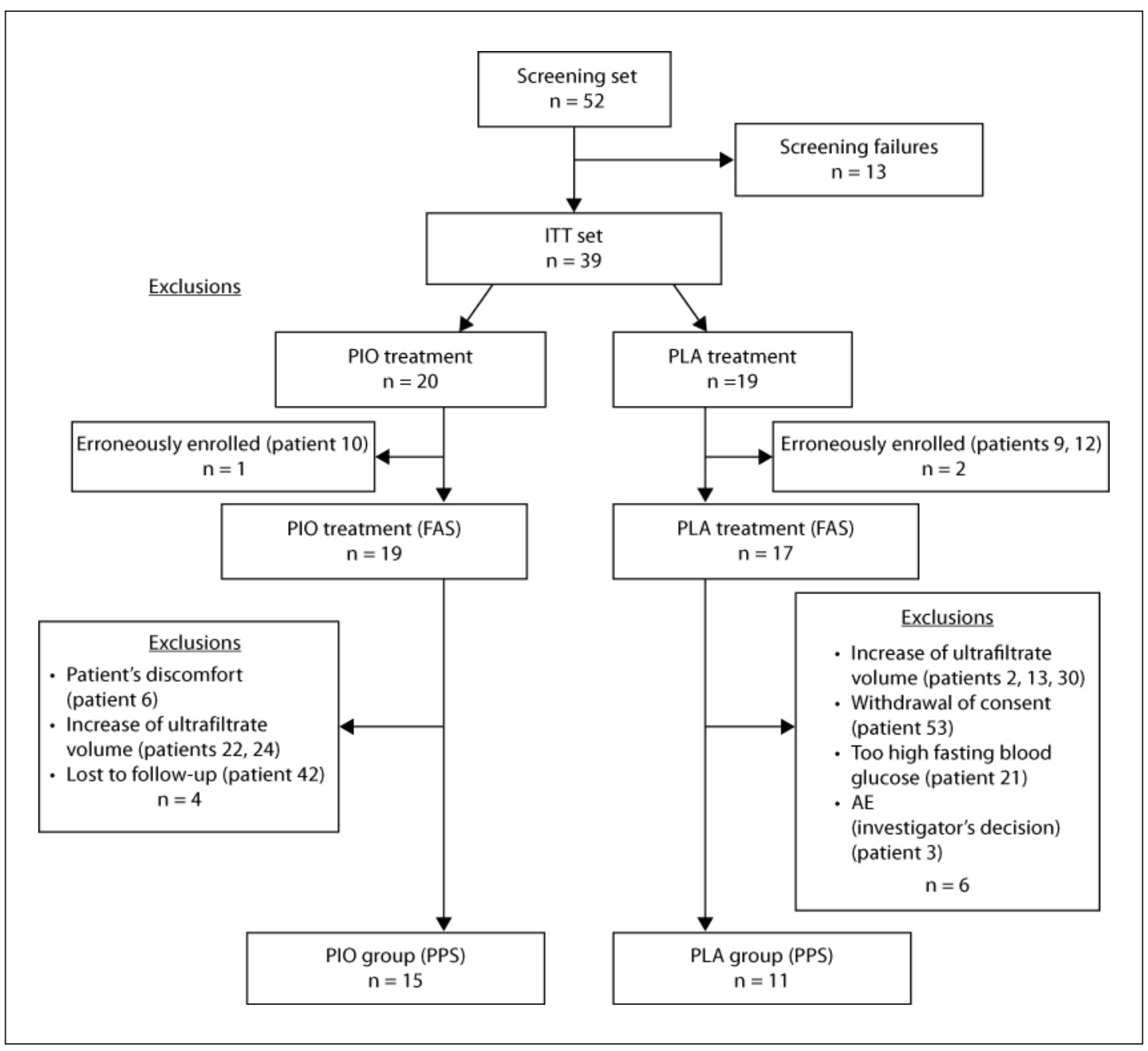

Fig. 2. Flowchart with numbers of patients that were screened, randomized, and assigned to the two treatment arms and treated accordingly (FAS) without major protocol violation as stipulated by the protocol (PPS).

\section{Demographics and Baseline Characteristics}

The most relevant demographic and baseline characteristics of patients belonging to the ITT set are summarized in table 1. These characteristics are corresponding for patients of both treatment groups. Consequently, the composition of both treatment groups represents a sound basis for a meaningful evaluation of the obtained data.

\section{Efficacy Endpoints}

Efficacy variables were analyzed for the FAS. The primary efficacy variable was the change of the total daily insulin dose after 6 months of treatment (V7) as compared to the baseline visit (V2). The mean daily insulin dosage at baseline was larger in the PIO group as compared to the PLA group. In the PIO group, mean daily insulin dose was reduced by $35 \%$ (from $63.96 \pm 48.90$ to $41.72 \pm 33.97 \mathrm{IU}$ ), while a decrease by $10 \%$ could be seen in the PLA arm (from $55.37 \pm 32.96$ to $49.95 \pm 29.33$ IU; table 2). Non-parametric Wilcoxon rank test indicated that the reduction in the total daily insulin requirements for the PIO group versus the PLA group was statistically significant $(\mathrm{p}=0.003)$. 
Table 1. Demographic and baseline characteristics of patients of both treatment groups for the ITT set (except for ultrafiltrate volumes, which are given for the FAS)

\begin{tabular}{lcc}
\hline & PIO & PLA \\
\hline Patients & 20 & 19 \\
Male/female & $14 / 6$ & $13 / 6$ \\
Age, years & $68.9 \pm 6.8$ & $69.6 \pm 9.4$ \\
Diabetes duration, years & $13.8 \pm 9.8$ & $12.4 \pm 8.2$ \\
BMI & $31.5 \pm 4.0$ & $30.3 \pm 4.6$ \\
Systolic blood pressure, mm Hg & $143.4 \pm 19.5$ & $144.6 \pm 22.8$ \\
Diastolic blood pressure, mm Hg & $72.7 \pm 13.3$ & $73.2 \pm 12.3$ \\
Ultrafiltrate volume, ml per dialysis session & $2,504 \pm 855$ & $2,743 \pm 999$ \\
\hline
\end{tabular}

Data are numbers or mean $\pm \mathrm{SD}$.

Table 2. Observation parameters at baseline and endpoint at 6 months

\begin{tabular}{|c|c|c|c|c|}
\hline & \multicolumn{2}{|l|}{$\mathrm{PIO}$} & \multicolumn{2}{|l|}{ PLA } \\
\hline & baseline & 6 months & baseline & 6 months \\
\hline Daily insulin dose, IU & $63.96 \pm 48.90$ & $41.72 \pm 33.97^{*}$ & $55.37 \pm 32.96$ & $49.95 \pm 29.33^{*}$ \\
\hline Daily insulin dose reduction $>30 \%, \mathrm{n}$ & 0 & 8 & 0 & 1 \\
\hline $\begin{array}{l}\text { HbAlc, } \% \\
\text { Mean change vs. baseline } \pm S D\end{array}$ & $7.4 \pm 0.9$ & $\begin{array}{r}6.8 \pm 1.0^{*} \\
-0.60 \pm 0.87\end{array}$ & $7.7 \pm 0.9$ & $\begin{array}{r}7.9 \pm 1.0^{*} \\
0.21 \pm 1.10\end{array}$ \\
\hline $\begin{array}{l}\text { Glucose, } \mathrm{mg} / \mathrm{dl} \\
\text { Mean change vs. baseline } \pm \mathrm{SD}\end{array}$ & $152.5 \pm 45.0$ & $\begin{array}{c}115.5 \pm 44.3 \\
-36.96 \pm 70.53\end{array}$ & $156.6 \pm 43.6$ & $\begin{array}{l}172.1 \pm 81.7 \\
15.51 \pm 68.61\end{array}$ \\
\hline
\end{tabular}

An insulin reduction of $\geq 30 \%$ was defined as a positive response to treatment and evaluated as a secondary efficacy variable. As depicted in table 2, 8 patients (out of 18,44\%) in the PIO group and 1 patient (out of 13,8\%) in the PLA group reached such a decrease in the daily insulin dose after 6 months. Comparison of HbAlc values at baseline and after 6 months demonstrates a statistically significant decrease of $8.08 \%$ in the PIO group ( $\mathrm{p}=$ 0.015 ), whereas in the PLA group the values increased by $2.68 \%$ (n.s.). Comparison between treatment groups indicates that the change in the PIO versus the PLA group is statistically significant with a p value of 0.004 (table 2). PIO treatment resulted in a reduction of the fasting blood glucose levels most pronounced at 6 months (-24.24\%) in comparison to baseline. Contrarily, blood glucose levels in the PLA group increased (9.9\%) at 6 months versus baseline (table 2). However, between-treatment-group confidence intervals and Student's tests demonstrate that this reduction in blood glucose is not statistically significant.

As for control of the cardiovascular risk profile several parameters concerning lipid metabolism and inflammation were analyzed. Changes of these secondary efficacy parameters after 6 months versus baseline are displayed in table 3.

$\mathrm{PIO}$ administration resulted in a pronounced decrease of the level of triglycerides $(-30 \%)$, while an increase was observed for the PLA group (19\%). HDL levels increased slightly in the PIO group (5\%), whereas they declined in the PLA group (-23\%). Total cholesterol levels decreased in both treatment groups (PIO $-7 \%$, PLA $-21 \%)$. LDL values were only reduced in 
Table 3. Changes of the secondary efficacy parameters between endpoint at 6 months and baseline

\begin{tabular}{|c|c|c|c|c|c|c|}
\hline \multirow[t]{3}{*}{ Secondary efficacy parameter } & \multicolumn{6}{|c|}{ Changes V7 (endpoint) vs. V2 (baseline) } \\
\hline & \multicolumn{3}{|c|}{$\mathrm{PIO}$} & \multicolumn{3}{|c|}{ PLA } \\
\hline & $\mathrm{n}$ & mean $\pm \mathrm{SD}$ (median) & change, $\%$ & $\mathrm{n}$ & mean $\pm \mathrm{SD}$ (median) & change, $\%$ \\
\hline Adiponectin, $\mu \mathrm{g} / \mathrm{ml}$ & 18 & $7.33 \pm 4.80(8.06)$ & 79.62 & 13 & $-1.37 \pm 2.56(-1.09)$ & -14.02 \\
\hline Fetuin A, g/l & 18 & $-0.04 \pm 0.12(-0.02)$ & -6.88 & 13 & $-0.09 \pm 0.20(-0.03)$ & -14.62 \\
\hline hs-CRP, mg/l & 13 & $0.63 \pm 1.54(0.91)$ & 20.08 & 7 & $0.49 \pm 0.70(1.17)$ & 12.66 \\
\hline Cholesterol, mg/dl & 13 & $-14.86 \pm 47.90(14.50)$ & -7.25 & 7 & $-40.61 \pm 71.87(-24.00)$ & -20.7 \\
\hline $\mathrm{HDL}, \mathrm{mg} / \mathrm{dl}$ & 13 & $1.47 \pm 6.07(1.00)$ & 4.72 & 7 & $-7.81 \pm 8.58(-3.00)$ & -22.9 \\
\hline LDL, mg/dl & 13 & $4.21 \pm 38.32(15.50)$ & 4.35 & 7 & $-35.14 \pm 46.68(-32.00)$ & -32.54 \\
\hline Triglycerides, mg/dl & 13 & $-107.75 \pm 140.21(-101.00)$ & -30.24 & 7 & $45.38 \pm 110.95(1.00)$ & 19.15 \\
\hline MMP-9, ng/ml & 18 & $89.14 \pm 548.68(-26.69)$ & 13.13 & 13 & $193.84 \pm 594.80(237.28)$ & 23.79 \\
\hline $\mathrm{MCP}-1, \mathrm{pg} / \mathrm{ml}$ & 18 & $-9.58 \pm 129.09(-5.08)$ & -1.69 & 13 & $300.0 \pm 978.58(52.10)$ & 46.83 \\
\hline E-selectin, $\mathrm{ng} / \mathrm{ml}$ & 18 & $-7.4 \pm 13.93(-7.98)$ & -16.27 & 13 & $6.81 \pm 13.73(6.43)$ & 14.73 \\
\hline Intact parathyroid hormone, ng/l & 18 & $-87.35 \pm 420.64(-12.00)$ & -26.9 & 13 & $85.61 \pm 142.99(90.00)$ & 31.3 \\
\hline NT-proBNP, pg/ml & 18 & $2,952.18 \pm 15,223.51(-350.00)$ & 57.5 & 13 & $5,273.61 \pm 14,495.30(996.00)$ & 104.38 \\
\hline Ultrafiltrate volume, $\mathrm{ml}$ & 18 & $113.48 \pm 617.00(202.45)$ & 4.53 & 14 & $361.71 \pm 567.72(483.30)$ & 13.19 \\
\hline Relaxin, pg/ml & 18 & $-4.11 \pm 37.54(-1.60)$ & -16.95 & 13 & $-5.70 \pm 31.93(-6.86)$ & -21.14 \\
\hline Carbonyl protein, $\mathrm{nmol} / \mathrm{ml}$ & 18 & $2.77 \pm 7.31(1.47)$ & 24.3 & 13 & $0.46 \pm 6.66(0.82)$ & 2.97 \\
\hline Angiotensin, pmol/l & 18 & $-4.41 \pm 34.49(3.52)$ & -17.25 & 13 & $5.02 \pm 43.34(-1.98)$ & 21.49 \\
\hline Myeloperoxidase, ng/ml & 18 & $-15.24 \pm 111.78(-2.13)$ & -20.28 & 13 & $8.05 \pm 36.43(8.00)$ & 17.97 \\
\hline Matrix Gla protein, nmol/l & 18 & $0.12 \pm 4.19(2.59)$ & 0.83 & 13 & $-1.40 \pm 5.34(-0.32)$ & -9.44 \\
\hline ox LDL, ng/ml & 13 & $41.08 \pm 23.29(0)$ & 69.64 & 7 & $-17.98 \pm 23.39(0)$ & -26.4 \\
\hline
\end{tabular}

The number of patients $(\mathrm{n})$ as well as absolute (mean \pm SD, median) and percentage changes are depicted for the FAS of both treatment groups.

the PLA group (-33\%), whereas they were increased in the PIO group (4\%). Finally, adiponectin levels were increased by PIO (80\%), and decreased by PLA (-14\%). However, among all the differences described only the change in adiponectin levels reached statistical significance for between- $(\mathrm{p}=0.001)$ and in-group comparison $(\mathrm{p}<0.001)$.

Concerning parameters used for evaluation of arterial inflammation, the level of the endothelial inflammation marker E-selectin was reduced by PIO administration ( $-16 \%)$, whereas it was increased in the PLA group (15\%). The change was statistically significant for within- $(p=0.029)$ and between-group comparison $(p=0.014)$. MCP-1 levels were almost unchanged upon PIO administration, but considerably increased in the PLA group. As for MMP-9 levels, no clear trend is obvious for either treatment group. No treatment-emergent effect of PIO was observed in the level of hs-CRP. The level of ox LDL was strongly increased in the PIO group and reduced in the PLA group. Carbonyl protein levels were increased in the PIO group, while in the PLA group, they were almost unchanged. Levels of fetuin A and relaxin showed the same tendencies to a slight reduction in both treatment groups. Unchanged or slightly reduced levels in both treatment groups were observed for the matrix Gla protein. For intact parathyroid hormone, a trend towards a decreased level in the PIO group and an increase in the PLA group was observed.

Of the cardiac risk markers analyzed, levels of myeloperoxidase increased in the PIO group during the study at V5 but were reduced compared to baseline after 6 months of treatment. In the PLA group, myeloperoxidase levels increased. NT-proBNP showed a trend towards a higher level in the PLA group in comparison to the PIO group, but levels fluctuated very strongly during the study. However, the differences mentioned above did not reach statistical significance.

While ultrafiltrate volumes of the PIO group fluctuated in the range of 2,504 $\mathrm{ml}$ at baseline and 2,618 $\mathrm{ml}$ at 6 months, those of the PLA group varied from 2,743 $\mathrm{ml}$ at baseline to 
Table 4. Number of AEs in both treatment groups and their intensity, classified and sorted according to system organ class (SOC) for the ITT set

\begin{tabular}{|c|c|c|c|c|c|c|c|c|c|c|c|}
\hline \multirow[t]{3}{*}{ SOC } & \multirow[t]{3}{*}{ Total } & \multicolumn{5}{|l|}{$\mathrm{PIO}$} & \multicolumn{5}{|l|}{ PLA } \\
\hline & & \multicolumn{4}{|c|}{ intensity of AE } & \multirow[t]{2}{*}{ total } & \multicolumn{4}{|c|}{ intensity of $\mathrm{AE}$} & \multirow[t]{2}{*}{ total } \\
\hline & & mild & mod. & sev. & NA & & mild & mod. & sev. & NA & \\
\hline Gastrointestinal disorders & 20 & 6 & 1 & 0 & 0 & 7 & 10 & 3 & 0 & 0 & 13 \\
\hline Investigations & 18 & 7 & 2 & 0 & 0 & 9 & 3 & 5 & 1 & 0 & 9 \\
\hline Infections and infestations & 17 & 4 & 4 & 0 & 0 & 8 & 6 & 3 & 0 & 0 & 9 \\
\hline Musculoskeletal and connective tissue disorders & 17 & 5 & 3 & 0 & 0 & 8 & 5 & 3 & 1 & 0 & 9 \\
\hline Injury, poisoning and procedural complications & 14 & 3 & 5 & 0 & 0 & 8 & 3 & 2 & 1 & 0 & 6 \\
\hline Metabolism and nutrition disorders & 11 & 5 & 0 & 0 & 0 & 5 & 4 & 2 & 0 & 0 & 6 \\
\hline Vascular disorders & 9 & 1 & 4 & 1 & 0 & 6 & 0 & 2 & 1 & 0 & 3 \\
\hline Cardiac disorders & 7 & 1 & 1 & 0 & 0 & 2 & 3 & 2 & 0 & 0 & 5 \\
\hline Nervous system disorders & 4 & 2 & 0 & 0 & 0 & 2 & 2 & 0 & 0 & 0 & 2 \\
\hline Skin and subcutaneous tissue disorders & 4 & 2 & 0 & 0 & 0 & 2 & 2 & 0 & 0 & 0 & 2 \\
\hline General disorders and administration site conditions & 3 & 0 & 0 & 0 & 0 & 0 & 2 & 0 & 1 & 0 & 3 \\
\hline Eye disorders & 2 & 0 & 2 & 0 & 0 & 2 & 0 & 0 & 0 & 0 & 0 \\
\hline Surgical and medical procedures & 2 & 0 & 1 & 0 & 0 & 1 & 1 & 0 & 0 & 0 & 1 \\
\hline Blood and lymphatic system disorders & 1 & 1 & 0 & 0 & 0 & 1 & 0 & 0 & 0 & 0 & 0 \\
\hline Total & 129 & 37 & 23 & 1 & 0 & 61 & 41 & 22 & 5 & 0 & 68 \\
\hline
\end{tabular}

mod. = Moderate; sev. = severe; NA = not available.

$3,105 \mathrm{ml}$ at 6 months. The ultrafiltrate volumes in the PLA group increased by $13.19 \% \mathrm{com}$ pared to $4.53 \%$ in the PIO group. The difference, however, was not statistically significant.

No statistically significant effects of PIO treatment on vital signs (weight, BMI, waist and hip circumference, blood pressure, and pulse) were observed in this study.

\section{Safety Analysis}

The ITT set was used to address safety issues. In total, 129 AEs occurred in the ITT set of this clinical trial. A slightly higher number of AEs was reported for patients of the PLA group $(n=68)$ than for those of the PIO group $(n=61)$. As summarized in table 4 , the majority of the AEs were gastrointestinal disorders, additional investigations, infectious disorders, and musculosceletal and connective tissue disorders. Most AEs were classified as 'mild' (PIO: 60.7\%; PLA: 60.3\%) and 'moderate' (PIO: 37.7\%; PLA: 32.4\%). AEs graded as 'severe' were observed once in the PIO group and 5 times in the PLA group.

The vast majority of AEs $(\mathrm{n}=109 ; 84.5 \%)$ was classified as unlikely or not related to treatment (PIO: $\mathrm{n}=51$; PLA: $\mathrm{n}=58$ ). Only 1 moderate AE in the PLA group was judged to be definitely related to treatment. Eight AEs (PIO: $n=3$; PLA: $n=5$ ) were assessed as probably caused by treatment.

A major focus of the safety evaluation was the occurrence of hypoglycemic episodes under combination treatment of PIO with insulin. There were 5 cases of hypoglycemia (all mild in nature) reported from 2 patients in the PIO group, and in the PLA group 2 patients suffered from 3 cases of hypoglycemia ( 1 mild and 2 moderate).

\section{Discussion}

The aim of the present study was to compare the effect of PIO versus PLA when administered in addition to insulin in patients with type 2 diabetes mellitus and renal failure requiring hemodialysis. For this purpose, 39 patients were randomized to the two treatment 
arms. One patient of the PIO group and 2 patients of the PLA group were early dropouts. Therefore, the FAS was made up of 19 and 17 patients treated with either PIO or PLA, respectively (fig. 1).

Evaluation of the primary efficacy variable after 6 months of treatment indicates a beneficial effect of PIO administration on insulin requirements (table 2). The low number of patients and the resultant fluctuation of the values impaired statistical confirmation of this result. Therefore, parametric ANCOVA and Student's t test could not be reliably applied because of violations of normal distribution and variance of the values. Use of the non-parametric Wilcoxon rank test revealed that PIO intake resulted in a statistically significant reduction of insulin requirements in patients with type 2 diabetes mellitus and renal failure as compared to patients receiving PLA.

The reduced insulin requirement went in parallel with an improvement in the secondary efficacy parameters regarding glycemic control (table 2). A statistically significant higher number of patients of the PIO group reached a $>30 \%$ reduction of the daily insulin dose after 6 months of treatment. The HbAlc value showed a statistically significant reduction in the PIO group, while it remained almost constant in the PLA group.

Lipids were differently affected by the two treatments (table 3). In contrast to PLA, PIO administration resulted in a beneficial effect regarding triglyceride and HDL levels. Total cholesterol levels were decreased in both treatment groups, whereas LDL levels were only reduced in the PLA group. Adiponectin levels were increased statistically significant in the PIO group, whereas they decreased in the PLA group. An increase of adiponectin upon PIO administration was previously reported and may be related to improved insulin resistance [20]. Reduced levels of triglycerides as well as slightly higher HDL levels may indicate a potential beneficial effect of PIO on atherosclerotic events. With respect to these positive effects, the statistically significant reduced level of the endothelial inflammation marker E-selectin in the PIO group is noteworthy (table 3). In line with these findings is the reduced amount of MCP-1 in the PIO group (table 3). These beneficial effects of PIO on E-selectin and MCP-1 were previously described for type 2 diabetes mellitus patients without renal failure, too [21]. Among the other atherosclerotic risk markers analyzed, hs-CRP and ox LDL have to be pointed out. No treatment-emergent effect of PIO was observed on the level of hs-CRP (table 3), deviating from the usually found decrease of hs-CRP due to PIO application in type 2 diabetes mellitus patients with normal renal function $[13,20]$. The level of ox LDL, a marker for oxidative stress, was strongly increased in the PIO group (table 3).

The effect of PIO administration on levels of myeloperoxidase was a mbiguously showing an increase during the study, but a reduction after 6 months of treatment (table 3). Although the levels of NT-proBNP fluctuated very strongly, a trend towards a lower level in the PIO group was observable (table 3). Average values of ultrafiltrate volumes of 6 consecutive dialyses prior to individual visits were calculated and increases $>30 \%$ in comparison to baseline were considered as worsening of heart insufficiency. Five patients (PIO: $\mathrm{n}=2$; PLA: $\mathrm{n}=$ 3) of the FAS exhibited a stronger increase and therefore had to discontinue study participation. The vast majority did not show a pronounced increase in the ultrafiltrate volume and, thus, was apparently not affected by worsening of heart insufficiency. Generally, ultrafiltrate volumes increased slightly stronger in the PLA group versus the PIO group during the course of the study (table 3). These findings indicate the absence of an adverse effect of PIO administration on heart function in patients of this study.

To date, only few reports exist in the literature about the effects of PIO in hemodialysis patients with type 2 diabetes mellitus. In general, they show that PIO is effective in glycemic control and well tolerated in that particular patient group [22-24]. However, these reports are well in line with the results of the present trial. In conclusion, this prospective, randomized, parallel, double-blind, PLA-controlled multi-center phase II trial showed that addition 
of PIO to insulin is well tolerated in patients with type 2 diabetes mellitus requiring hemodialysis. No indications for a volume overload accompanied by symptoms of heart failure and no indications for a hypoglycemia risk were found with this treatment. In fact, addition of PIO showed an improved glycemic control with a simultaneous significant reduction in daily insulin requirements.

\section{Conclusion}

Addition of PIO to insulin in patients with type 2 diabetes requiring hemodialysis resulted in a decrease of the daily insulin dose and an improved glycemic control. Furthermore, PIO administration was well tolerated without any indications of volume overload and negative influence on hypoglycemia risk.

\section{Acknowledgment}

The trial has been sponsored by TAKEDA Pharma GmbH, Aachen, Germany.

\section{References}

1 Centers for Disease Control and Prevention (CDC): Incidence of end-stage renal disease among persons with diabetes - United States, 1990-2002. MMWR 2005;54:1097-1100.

2 The effect of intensive treatment of diabetes on the development and progression of long-term complications in insulin-dependent diabetes mellitus. The Diabetes Control and Complications Trial Research Group. N Engl J Med 1993;329:977-986.

-3 Stratton IM, Adler AI, Neil HA, et al: Association of glycaemia with macrovascular and microvascular complications of type 2 diabetes (UKPDS 35): prospective observational study. BMJ 2000;321: 405-412.

-4 Iglesias P, Diez JJ: Peroxisome proliferator-activated receptor gamma agonists in renal disease. Eur J Endocrinol 2006;154:613-621.

-5 Yale JF: Oral antihyperglycemic agents and renal disease: new agents, new concepts. J Am Soc Nephrol 2005;16(suppl 1):S7-S10.

-6 Czock D, Aisenpreis U, Rasche FM, Jehle PM: Pharmacokinetics and pharmacodynamics of lisproinsulin in hemodialysis patients with diabetes mellitus. Int J Clin Pharmacol Ther 2003;41:492-497.

-7 Filiopoulos V, Vlassopoulos D: Inflammatory syndrome in chronic kidney disease: pathogenesis and influence on outcomes. Inflamm Allergy Drug Targets 2009;8:369-382.

-8 Kasiske BL: The kidney in cardiovascular disease. Ann Intern Med 2001;134:707-709.

-9 Hunsicker LG, Adler S, Caggiula A, et al: Predictors of the progression of renal disease in the Modification of Diet in Renal Disease Study. Kidney Int 1997;51:1908-1919.

10 Knight EL, Rimm EB, Pai JK, et al: Kidney dysfunction, inflammation, and coronary events: a prospective study. J Am Soc Nephrol 2004;15:1897-1903.

- 11 Clausen P, Jensen JS, Jensen G, Borch-Johnsen K, Feldt-Rasmussen B: Elevated urinary albumin excretion is associated with impaired arterial dilatory capacity in clinically healthy subjects. Circulation 2001;103:1869-1874.

-12 Shah P, Mudaliar S: Pioglitazone: side effect and safety profile. Expert Opin Drug Saf 2010;9:347-354.

-13 Rizos CV, Liberopoulos EN, Mikhailidis DP, Elisaf MS: Pleiotropic effects of thiazolidinediones. Expert Opin Pharmacother 2008;9:1087-1108.

-14 Pfutzner A, Schneider CA, Forst T: Pioglitazone: an antidiabetic drug with cardiovascular therapeutic effects. Expert Rev Cardiovasc Ther 2006;4:445-459. 
15 Pfützner A, Weber MM, Forst T: Pioglitazone: update on an oral antidiabetic drug with antiatherosclerotic effects. Expert Opin Pharmacother 2007;8:1985-1998.

-16 Dormandy JA, Charbonnel B, Eckland DJ, et al: Secondary prevention of macrovascular events in patients with type 2 diabetes in the PROactive Study (PROspective pioglitAzone Clinical Trial In macroVascular Events): a randomised controlled trial. Lancet 2005;366:1279-1289.

-17 Erdmann E, Dormandy JA, Charbonnel B, Massi-Benedetti M, Moules IK, Skene AM: The effect of pioglitazone on recurrent myocardial infarction in 2,445 patients with type 2 diabetes and previous myocardial infarction: results from the PROactive (PROactive 05) Study. J Am Coll Cardiol 2007;49: 1772-1780.

-18 Wilcox R, Bousser MG, Betteridge DJ, et al: Effects of pioglitazone in patients with type 2 diabetes with or without previous stroke: results from PROactive (PROspective pioglitAzone Clinical Trial In macroVascular Events 04). Stroke 2007;38:865-873.

-19 Schneider CA, Ferrannini E, Defronzo R, Schernthaner G, Yates J, Erdmann E: Effect of pioglitazone on cardiovascular outcome in diabetes and chronic kidney disease. J Am Soc Nephrol 2008;19:182187.

-20 Pfutzner A, Hohberg C, Lubben G, et al: Pioneer study: PPARgamma activation results in overall improvement of clinical and metabolic markers associated with insulin resistance independent of long-term glucose control. Horm Metab Res 2005;37:510-515.

-21 Pfützner A, Marx N, Lubben G, et al: Improvement of cardiovascular risk markers by pioglitazone is independent from glycemic control: results from the pioneer study. J Am Coll Cardiol 2005;45:19251931.

-22 Abe M, Okada K, Kikuchi F, Matsumoto K: Clinical investigation of the effects of pioglitazone on the improvement of insulin resistance and blood pressure in type 2-diabetic patients undergoing hemodialysis. Clin Nephrol 2008;70:220-228.

23 Abe M, Kikuchi F, Kaizu K, Matsumoto K: Combination therapy of pioglitazone with voglibose improves glycemic control safely and rapidly in Japanese type 2-diabetic patients on hemodialysis. Clin Nephrol 2007;68:287-294.

-24 Manley HJ, Allcock NM: Thiazolidinedione safety and efficacy in ambulatory patients receiving hemodialysis. Pharmacotherapy 2003;23:861-865. 